\title{
Identification of the hub genes and transcription factor-miRNA axes involved in Helicobacter pylori-associated gastric cancer
}

\author{
PENG ZHAO $^{1 *}$, XIANXIONG MA $^{2 *}$, JIANCHENG CHENG $^{3 *}$, HENGYU CHEN $^{4,5}$ and LEI LI ${ }^{6}$ \\ Departments of ${ }^{1}$ Hepatobiliary Surgery and ${ }^{2}$ Gastrointestinal Surgery, Union Hospital, Tongji Medical College, \\ Huazhong University of Science and Technology; ${ }^{3}$ Department of Cardiovascular Surgery, Union Hospital, \\ Tongji Medical College, Huazhong University of Science and Technology, Wuhan, Hubei 430022; \\ ${ }^{4}$ Department of Breast and Thyroid Surgery, The Second Affiliated Hospital of Hainan Medical University, Haikou, \\ Hainan 570100; ${ }^{5}$ Department of Pancreatic Surgery, Union Hospital, Tongji Medical College; \\ ${ }^{6}$ Department of Breast and Thyroid Surgery, Union Hospital, Tongji Medical College, \\ Huazhong University of Science and Technology, Wuhan, Hubei 430022, P.R. China
}

Received February 5, 2020; Accepted November 26, 2021

DOI: 10.3892/ol.2022.13209

\begin{abstract}
It has been previously reported that transcription factor-microRNA (TF-miRNA) axes play a significant role in the carcinogenesis of several types of malignant tumor. However, there is a lack of research into the differences in the mechanism of Helicobacter pylori (HP)-positive [ $\mathrm{HP}(+)]$ and HP-negative $[\mathrm{HP}(-)]$ gastric cancer. The aim of the present study was to identify the hub genes and TF-miRNA axes, and to determine the potential mechanisms involved in HP-associated gastric cancer. HP-associated mRNA and miRNA data, as well as the corresponding clinical information, was downloaded from The Cancer Genome Atlas database. Differentially expressed genes (DEGs) and DE miRNAs (DEMs) were then identified from the $\mathrm{HP}(+)$ and $\mathrm{HP}(-)$ cancer
\end{abstract}

Correspondence to: Dr Hengyu Chen, Department of Breast and Thyroid Surgery, The Second Affiliated Hospital of Hainan Medical University, 368 Yehai Avenue, Haikou, Hainan 570100, P.R. China E-mail: chenhy9012@163.com

Dr Lei Li, Department of Breast and Thyroid Surgery, Union Hospital, Tongji Medical College, Huazhong University of Science and Technology, 1277 Jiefang Avenue, Wuhan, Hubei 430022, P.R. China

E-mail: leili2008@hust.edu.cn

*Contributed equally

Abbreviations: TF, transcription factor; TGGA, The Cancer Genome Atlas; PPI, protein-protein interaction; DEG, differentially expressed gene; DEM, differentially expressed microRNA; HP, Helicobacter pylori; GO, Gene Ontology; KEGG, Kyoto Encyclopedia of Genes and Genomes; STRING, Search Tool for the Retrieval of Interacting Genes

Key words: Helicobacter pylori, gastric cancer, transcription factor, microRNA, The Cancer Genome Atlas, bioinformatics
mRNA and miRNA datasets, respectively. Subsequently, gene set enrichment analysis and the protein-protein interaction (PPI) networks were investigated using the ClusterProfiler packages. Lastly, TF-miRNA-DEG networks were constructed using the miRWalk online tool. A total of 1,050 DEGs and 13 DEMs were identified from the normalized mRNA and miRNA expression datasets, respectively. In addition, 180 Gene Ontology terms and 30 Kyoto Encyclopedia of Genes and Genomes pathways were found to be enriched, while 6 hub genes were identified from the PPI analysis. Furthermore, 7 TF-miRNA interactions and 181 TF-miRNA-DEG axes were constructed using an integrated bioinformatics approach, while 2 TF-miRNA interactions (ZEB1-miRNA-144-3p and PAX2-miRNA-592) were confirmed using reverse transcription-quantitative PCR in samples from enrolled patients. Moreover, the ZEB1-miRNA-144-3p axis was further validated based on dual luciferase reporter assay results. In summary, an integrated bioinformatics approach was used to screen the significant molecular and regulatory axes, which may provide a novel direction to investigate the pathogenesis of gastric cancer associated with HP.

\section{Introduction}

Helicobacter pylori (HP) has been classified as a group I carcinogen by The International Agency for Research on Cancer (1). A previous study revealed that chronic gastritis and gastric ulcers were caused by HP, which may be a factor to increase the risk of gastric cancer (2). However, the exact mechanism of how HP is involved in the development of gastric cancer has not been elucidated.

MicroRNAs (miRNAs/miRs) are short non-coding RNAs that can regulate the translation of genes by binding to the 3'-untranslated regions of the target gene (3). miRNAs are involved in various important biological processes in the development of gastric cancer, such as cell proliferation, invasion and metastasis (4). Previous studies have found that miRNAs play significant roles in the regulation of tumor biology (5-7), 
and in the regulation of the physiology and pathology of the immune system (8). Some miRNAs serve as therapeutic targets or as prognostic biomarkers of gastric cancer $(9,10)$. For example, poor prognosis and advanced features in patients with non-small cell lung cancer could be predicted by the downregulation of miR-503 expression (11).

Transcription factors (TFs) are significant regulatory factors that can serve as oncogenes or tumor suppressors by binding to the target genes in the gene expression pathway $(12,13)$. TFs serve as regulators that act at the transcriptional level, while miRNAs function at the post-transcriptional level $(3,12)$. A large number of studies (14-16) have shown that transcriptional and post-transcriptional regulators of gene expression interact with each other in the molecular pathology of numerous diseases, such as the role of FBL in epithelial mesenchymal transition and cardiogenesis (17). miRNAs and TFs are regulated by each other in feedback loops (17). Previous studies have reported that miRNAs are regulated by TFs (TF-miRNA regulation); for example, the identification of a TF-miRNA network was found in esophageal adenocarcinoma using bioinformatics analysis (18). However, associations between the TF-miRNA-mRNA network and HP-associated gastric cancer have not been investigated. The aim of the present study was to identify the hub genes and TF-miRNA axes, and to identify the potential mechanisms involved in HP-associated gastric cancer.

\section{Materials and methods}

Patients and tissue specimens. A total of 30 tumor tissues [10 HP-negative (-) and $20 \mathrm{HP}$-positive (+)] were collected from patients with gastric cancer who underwent gastrectomy at the Department of Gastrointestinal Surgery, Union Hospital of Huazhong University of Science and Technology (Wuhan, China). All procedures were approved by the Ethics Committee of the Union Hospital of Huazhong University of Science and Technology, and conducted according to Declaration of Helsinki principles. Prior written and informed consent was obtained from each patient or their guardians. The clinicopathological information of the patients is shown in Table SI.

Data sourcing and processing. Genes and miRNA expression profiles, as well as the corresponding clinical information, from patients with gastric cancer, were downloaded from The Cancer Genome Atlas (TCGA) database using TCGA biolinks package (19). Samples containing clear HP infection information were included. Samples with unavailable HP infection information, samples of paracancer tissue and samples of normal tissue were removed. The HP status of the tissue samples from the enrolled patients was confirmed using a 13C-urea breath test with $75 \mathrm{mg}$ urea (UREA 13C breath test Heliforce kit; Beijing Richen-Force Science \& Technology Co. Ltd.). A total of 141 gastric samples [16 cases were HP(+) and 125 cases were $\mathrm{HP}(-)]$ were selected for the following bioinformatics analysis. The differentially expressed genes (DEGs) with significant differential expression between the $\mathrm{HP}(+)$ and $\mathrm{HP}(-)$ groups were selected. A gene would be selected using the following conditions: i) Expression level 1.2 times higher in $20 \%$ of the total number of samples compared with the median expression level in the total number of samples; and ii) the variance in the expression level in every sample was higher than the median level. Subsequently, $\log 2$ transformation and $\mathrm{Z}$ correction were performed to normalize the expression value of each gene.

Identification of DEGs and DE miRNAs (DEMs). Limma package (20) was applied to compute DEGs and DEMs based on the aforementioned normalized mRNA and miRNA expression data. Bayes test was used to identify DEGs with $\geq 2$ fold-change and a P-value cutoff of 0.05 was defined as statistically significant.

Gene Ontology (GO) and Kyoto Encyclopedia of Genes and Genomes (KEGG) pathway enrichment analysis of DEGs. Genes and gene products were annotated using GO analysis, which is a common and useful method for identifying characteristic biological attributes of transcriptome data or from high-throughput genomic data. KEGG serves as a knowledge base for systematic analysis of gene functions, and linkage between genomic information and higher-order functional information. Comprehensively mapping a set of genes to the associated biological annotation is an important foundation for the success of the gene functional analysis of any high-throughput data. KEGG pathway analysis and GO enrichment was performed using the ClusterProfiler package (21) to analyze the DEGs at the functional level. $\mathrm{P}<0.05$ was considered to indicate a statistically significant difference.

Construction and integration of the PPI network. The PPI network was investigated using the Search Tool for the Retrieval of Interacting Genes (STRING) online database (22). STRING (v11.0) was used, which covers 24,584,628 proteins from 5,090 organisms. The DEGs were mapped using the STRING database to determine the interactive associations among DEGs, and then, the experimentally validated interactions with a significant combined score $>0.4$ were selected for further analysis. PPI networks were then constructed with the CytoScape software (v3.6.1) (23). Subsequently, the sub-network modules were screened using the plug-in molecular complex detection (MCODE) in CytoScape. The following screening criteria was used: MCODE score $>3$ and node number $>6$. In addition, the function and pathway enrichment analysis of DEGs in the sub-module was also determined. $\mathrm{P}<0.05$ was considered to indicate a statistically significant difference.

Construction of TF-miRNA-gene axis. The TFs and DEMs were selected to construct TF-miRNA regulatory networks using the miRWalk2.0 database (24). The TF-miRNAs were identified as hub interactions, as predicted by the miRWalk 2.0 database, TargetScan6.2 database (25), miRanda database (26) and RNA22 database (27) simultaneously. The miRNA-DEG regulatory networks were constructed across the aforementioned method. Finally, TF-miRNA-gene axes were constructed to identify a transcriptional regulation model and reveal significant networks involved in HP-associated gastric cancer.

Cell culture. The gastric cancer MKN45 cell line was purchased from the American Type Culture Collection. The MNK-450 cells were cultured in RPMI 1640 medium (HyClone; Cytiva) 
with $10 \%$ FBS and $1 \%$ penicillin-streptomycin under a humidified atmosphere with $5 \% \mathrm{CO}_{2}$ at $37^{\circ} \mathrm{C}$.

Validation of TF and targeted DEMs. DE TFs and DEMs were confirmed using reverse transcription-quantitative PCR (RT-qPCR) in samples from enrolled patients. To confirm the results from the integrated bioinformatics analysis, 20 fresh $\mathrm{HP}(+)$ and 10 fresh $\mathrm{HP}(-)$ gastric cancer tissues were collected and examined by experienced pathologists at the Department of Gastrointestinal Surgery, Union Hospital of Huazhong University of Science and Technology between September 1 and December 30, 2019. The tissue samples were frozen and stored immediately in liquid nitrogen following surgical resection. Total cellular RNA was extracted from the gastric samples using RNAiso Plus (Takara Bio, Inc.). For miRNA quantification, synthesis of cDNA was performed using the PrimeScript $^{\mathrm{TM}}$ RT reagent kit (GeneCopoeia, Inc.) according to the manufacturer's protocols. The purity and concentration of RNA was measured using a Nanodrop2000 (Thermo Fisher Scientific, Inc.). qPCR was performed using the Real-time PCR Detection System (SLAN; Shanghai Hongshi Medical Technology, Co., Ltd.) and the SYBR Premix Ex Taq II kit (Takara Bio, Inc.). The conditions of PCR cycling were as following: Activation of TaqMan at $95^{\circ} \mathrm{C}$ for $10 \mathrm{~min}$, and then 40 cycles of denaturation at $95^{\circ} \mathrm{C}$ for $10 \mathrm{sec}$, and annealing/extension at $60^{\circ} \mathrm{C}$ for $60 \mathrm{sec}$. Relative miRNA expression levels were normalized to U6, while mRNA was normalized to GAPDH, and quantification was performed using the $2^{-\Delta \Delta \mathrm{Cq}}$ method (28). The primer sequences are shown in Table I. R language (https://www.r-project.org/) was used to analyze the RT-qPCR data. All reactions were performed in triplicate.

Western blot analysis. Tissue lysates were extracted using the RIPA lysis buffer (Beyotime Institute of Biotechnology) supplemented with $1 \%$ phenylmethylsulfonyl fluoride (Sigma-Aldrich; Merck KGaA) according to the manufacturer's instructions. The protein concentration was measured using the Pierce bicinchoninic acid protein assay kit (Thermo Fisher Scientific, Inc.). The same amount of protein $(30 \mu \mathrm{g})$ from all patients with gastric cancer was separated on $10 \%$ gels using SDS-PAGE (Bio-Rad Laboratories, Inc.) and transferred onto a PVDF membrane (EMD Millipore) After that, the membrane was blocked for $1 \mathrm{~h}$ with $5 \%$ non-fat milk and $1 \mathrm{X}$ TBST $(0.05 \%)$ at room temperature. Next, the primary antibodies were used to incubate the membrane at $4^{\circ} \mathrm{C}$ overnight followed by incubation with secondary antibodies for $2 \mathrm{~h}$ at room temperature. $\beta$-actin was used as a loading control. The following primary antibodies were used: Rabbit anti-ZEB1 (1:1,000; cat. no. 021544-1-AP; ProteinTech Group, Inc.), rabbit anti-LHX3 (1:1,000; cat. no. 20745-1-AP, ProteinTech Group, Inc.), rabbit anti-PAX2 (1:2,000; cat. no. ab79389; Abcam) and mouse anti- $\beta$-actin (1:5,000; cat. no. 60008-1-Ig; ProteinTech Group, Inc.). Anti-mouse IgG (1:5,000; cat. no. ab208001; Abcam) and anti-rabbit IgG (1:5,000; cat. no. ab207999; Abcam) were used as the secondary antibodies. Proteins bands were visualized using electrochemiluminescence (ECL Western Blotting Substrate; cat. no. 32106; Pierce; Thermo Fisher Scientific, Inc.) and analyzed using the ChemiDoc ${ }^{\mathrm{TM}}$ XRS Molecular Imager 3.0 system (Bio-Rad Laboratories, Inc.).
Table I. Sequences of reverse transcription-quantitative PCR primers.

Primers Sequences (5'-3')

\section{HIC1}

Forward

Reverse

GCGCCGCTGCTCCCCTCTTTGTG

LHX3

Forward ACCCAGGCCCGGCTCGTGCTTCAT

Reverse

GCCCAGCCCAGCCCAGCATAGC

LMX1B

Forward

GAGAAGGGGCGCCAGGCATTTTTG

Reverse

GGGGGTGCTGCTGGGCTCCGACTG

MAFB

Forward

Reverse

CCCCGCTGCCCTTGCTCTGACTGC

PAX2

Forward

Reverse

SLA2

Forward

Reverse

U6

Forward

Reverse

GCTCGGCGCCCAAATCTCATCAGT

CGGTTTGGCGGGGCGGGTATTTA

hsa-miR-144-3p

Forward

GGGCGCGGGCGGAGCACAC

GGGTAGGGGCCGGCCGTTCACAA

Reverse

hsa-miR-3176

Forward

Reverse

TCATCCGGGAGAGCCAGACCAG GGGCCTAGGCATCATCCAAAGA

hsa-miR-592

Forward

Reverse

hsa-miR-659

Forward

Reverse

CTCGCTTCGGCAGCACA

AACGCTTCACGAATTTGCGT

GCGCGCGACAGTATAGATGAT

CAGTGCGTGTCGTGGAGT

GCGACTGGCCTGGGAC

CAGTGCGTGTCGTGGAGT

ZEB1

Forward

Reverse

GCGCTTGTGTCAATATGCGA
CAGTGCGTGTCGTGGAGT

CAGTGCGTGTCGTGGAGT

GCGAGGACCTTCCCTGAAC

CAGTGCGTGTCGTGGAGT

GAPDH

Forward

Reverse

CGCAGTCTGGGTGTAATCGT

TTGCAGTTTGGGCATTCATA

TTAAAAGCAGCCCTGGTGAC TGTGGTCATGAGTCCTTCCA

Transfection. ZEB1-small interfering (si)RNA (siZEB1), PAX2-siRNA (siPAX2) and corresponding negative control (NC-siRNA) (Table II) were purchased from Guangzhou RiboBio Co., Ltd. ZEB1 and PAX2 pcDNA3.1 plasmid (ZEB1 and PAX2 overexpression plasmid) and the control vector were purchased from Shanghai GeneChem Co., Ltd. All siRNAs were transfected at a final concentration of $50 \mathrm{nmol} / \mathrm{l}$, and the plasmid was transfected at final concentration using $1.6 \mu \mathrm{g}$ for 
Table II. Sequences of siRNAs.

\begin{tabular}{lc}
\hline siRNA targets & \multicolumn{1}{c}{ Sequences (5'-3') } \\
\hline PAX2-siRNA & \\
Sense & CGACUAUGUUCGCCUGGGATT \\
Antisense & UCCCAGGCGAACAUAGUCGGG \\
ZEB1-siRNA & \\
Sense & GCGGCGCAAUAACGUUACAAA \\
Antisense & UGUAACGUUAUUGCGCCGCGG \\
NC-siRNA & \\
Sense & UAUAAGUGUGACUACUAACTT \\
Antisense & GUUAGUAGUCACACUUAUATT \\
\hline
\end{tabular}

siRNA, small interfering RNA; NC, negative control.

a 12-well plate. Lipofectamine ${ }^{\circledR} 3000$ (Invitrogen; Thermo Fisher Scientific, Inc.) was used for cell transfection at $37^{\circ} \mathrm{C}$ for $48 \mathrm{~h}$ according to the manufacturer's instructions and then subsequent experiments were immediately performed. The promoter reporter construct ( $\Delta \mathrm{E}$-box) was constructed with the QuikChange site-directed mutagenesis kit (Stratagene; Agilent Technologies, Inc.).

Luciferase reporter assay. The JASPAR database (http://jaspar.genereg.net) was used to predict the binding region of the miRNA-144 according to the manufacturer's protocols (29). To determine whether ZEB1 targeted the promoter region of the miR-144 gene, using Lipofectamine 3000 , the gastric adenocarcinoma MKN-45 cell line was transfected with the recombinant pGL4.1 plasmid (Promega Corporation), including the wild-type or mutant miR-144 gene promoter, empty vector, ZEB1 overexpression plasmid and siZEB1 and NC (siNC). The empty vector and siNC were used as the negative controls for the luciferase reporter gene assay. After $48 \mathrm{~h}$, firefly and Renilla luciferase activities were detected using the dual-luciferase reporter assay system (Promega Corporation). Renilla luciferase activity was used for normalization.

Statistical analysis. Statistical analyses were performed applying R software (4.0.0; https://www.r-project.org/) unless otherwise noted. All results are presented as the mean \pm SD and were assessed via Graphpad Prism 7 (Graphpad Software, Inc.). The significance of differences between two groups was evaluated via Student's unpaired t-test, and the comparison among multiple groups was conducted via one-way ANOVA followed by the LSD post hoc test. All experiments were performed 3 times. $\mathrm{P}<0.05$ was used to indicate a statistically significant difference.

\section{Results}

Identification of DEGs and DEMs. There were 24,991 gene and 1,881 miRNA expression profiles from 141 gastric cancer samples obtained from TCGA mRNA and miRNA datasets, respectively. A total of 1,050 genes and 13 miRNAs were found to be DE in the HP-associated gastric cancer samples, as shown in Fig. 1. The top 20 DEGs are shown in Fig. 2A, while the 13 DEMs are listed in Fig. 2B.

Gene set enrichment analysis. All of the 1,050 DEGs identified, which were based on the normalized mRNA expression data, were screened for gene set enrichment analysis. A total of 180 significant enrichments were identified using the GO analysis, including in the three main categories of biological process, cell component and molecular function. The top three GO terms were 'T cell activation', 'leukocyte proliferation' and 'leukocyte migration'. The top $12 \mathrm{GO}$ terms according to the P-values and the top $5 \mathrm{GO}$ enrichments with the gene linkages are displayed in Fig. 3A and B, respectively. Pathway analysis using the KEGG database found that these genes were significantly enriched in 'Staphylococcus aureus infection', 'systemic lupus erythematosus' and 'hematopoietic cell lineage'. The top 12 KEGG pathways and the top 5 pathways with the gene linkages are displayed in Fig. $3 \mathrm{C}$ and $\mathrm{D}$, respectively.

PPI network and sub-network analyses. The top 400 DEGs were imported into the STRING database to identify the interactive association between the proteins. Only the genes with a combined score $>0.4$ were selected to create the network. Finally, 1,434 pairs of protein associations were identified following removal of the unmatched genes. In addition, genes with $>60$ edges were considered as hub genes. A total of 6 significant genes were identified based the annotated information from the STRING database: TYROBP, CCR5, C3AR1, LCP2, CCL5 and SPI1 (Fig. 3E).

A total of 252 nodes and 1,434 edges were analyzed using the plug-in, MCODE. The top 2 significant modules were selected for the gene functional annotation involved in the modules. Enrichment analysis showed that the genes in those 2 modules were primarily associated with phytochelatin metabolic process and positive regulation of apoptosis (Fig. 3F).

Construction of the TF-miRNA-mRNA network. Using the mRNA and miRNA expression data from TCGA database, 1,050 DEGs and 13 DEMs were identified. From these, 31 DE TFs were identified from the Trrust database (Fig. 4A). Next, the regulatory associations were constructed using the $31 \mathrm{TFs}$ and 13 miRNAs and the miRWalk database, and finally, 7 TF-miRNA pairs (including 7 genes and 4 miRNAs) were constructed (Fig. 4B). Subsequently, 107 pairs of miRNA-DEG interactions were also constructed using the 4 miRNAs and 1,050 DEGs, and the miRWalk database (Fig. 4C). Combining the aforementioned regulatory associations, 181 TF-miRNA-DEG regulatory associations were finally identified (Fig. 4D) (Table III).

Confirmation using RT-qPCR and western blot analysis, and ZEB1-miRNA-144 axis validation. RT-qPCR was used to investigate the expression level of the 7 TF-miRNAs (Table III). It was revealed that the expression level of 3 genes and 2 miRNAs were dysregulated [Figs. 5 and 6; $\mathrm{HP}(+)$ is represented by the case group and HP(-) is represented by the control group]. Among these genes and miRNAs, 


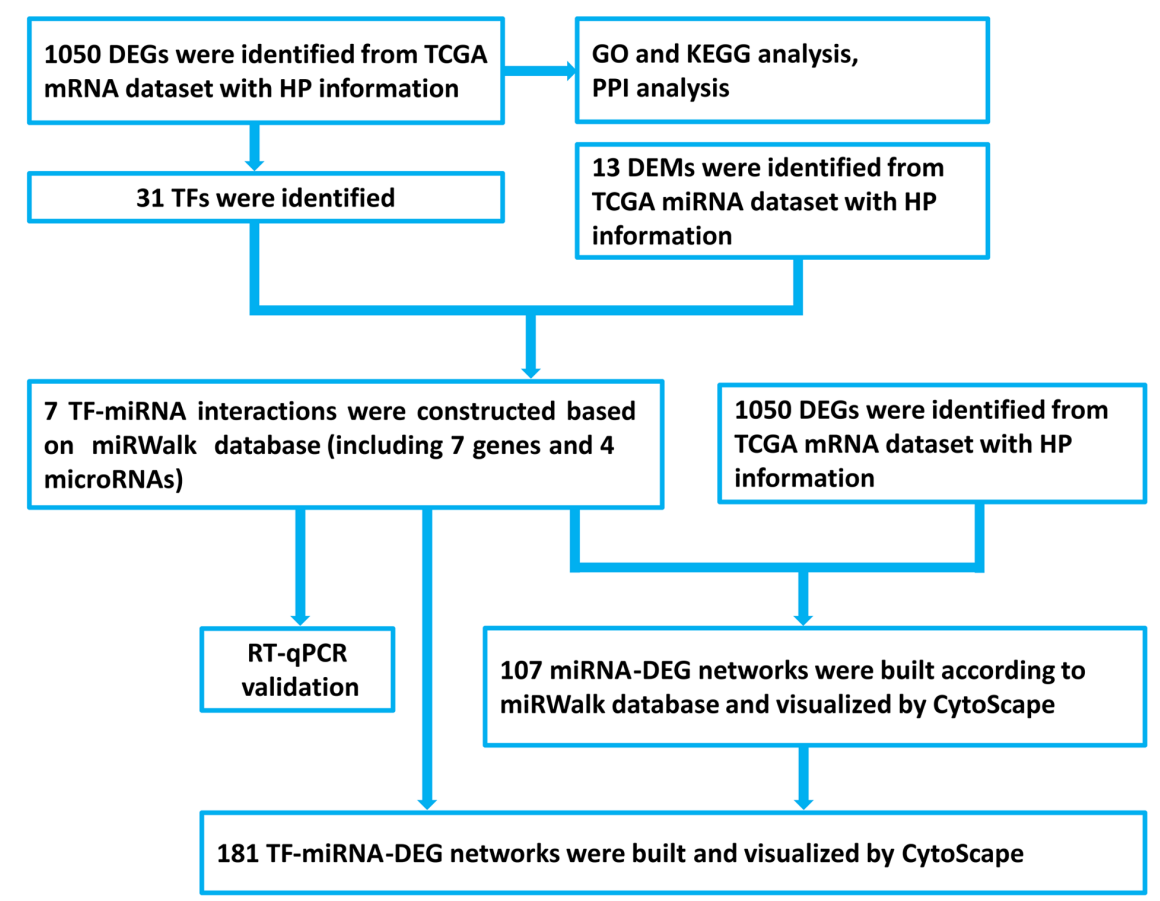

Figure 1. Flow chart of the bioinformatics analysis process. DEG, differentially expressed gene; TCGA, The Cancer Genome Atlas; GO, Gene Ontology; KEGG, Kyoto Encyclopedia of Genes and Genomes; PPI, protein-protein interaction; HP, Helicobacter pylori; TF, transcription factor; miRNA, microRNA; RT-qPCR, reverse transcription-quantitative PCR.

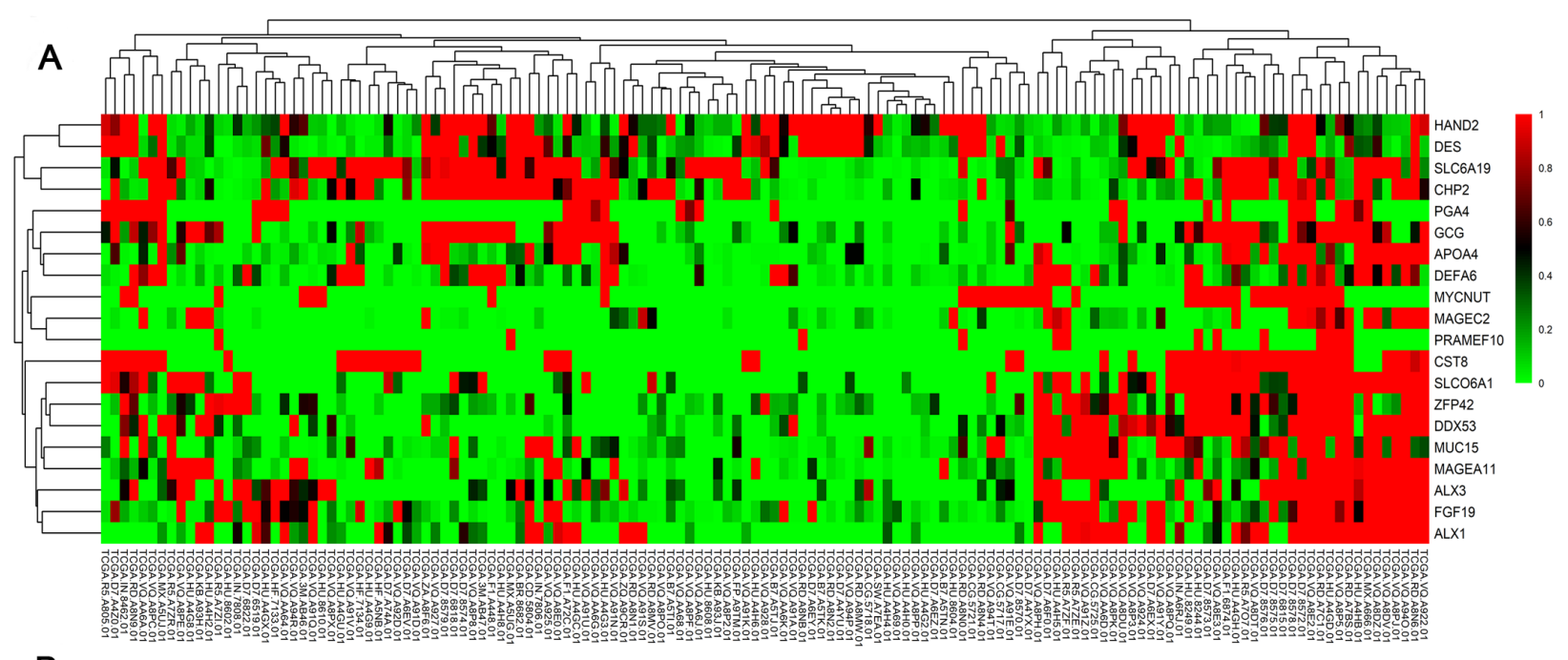

B

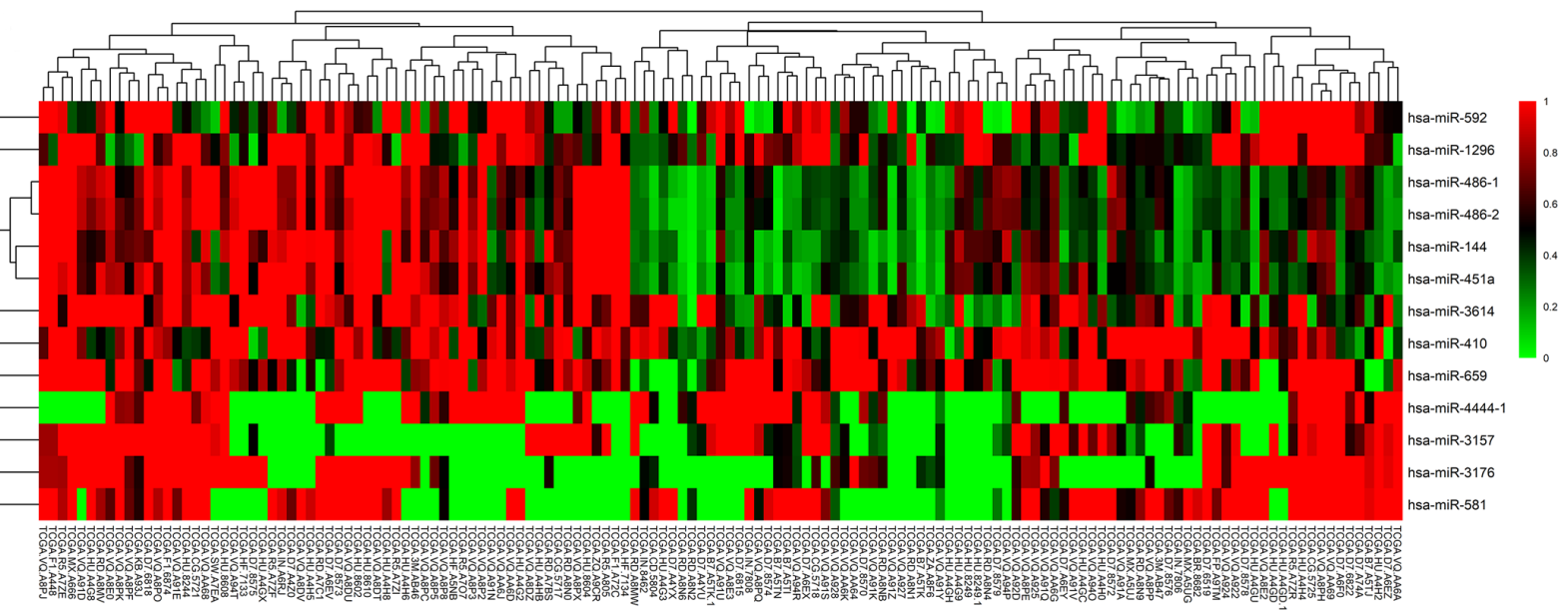

Figure 2. Expression heatmap of top (A) 20 differentially expressed genes and (B) 13 differentially expressed miRNAs based on The Cancer Genome Atlas Helicobacter pylori-associated mRNA and miRNA expression data. miRNA, microRNA. 

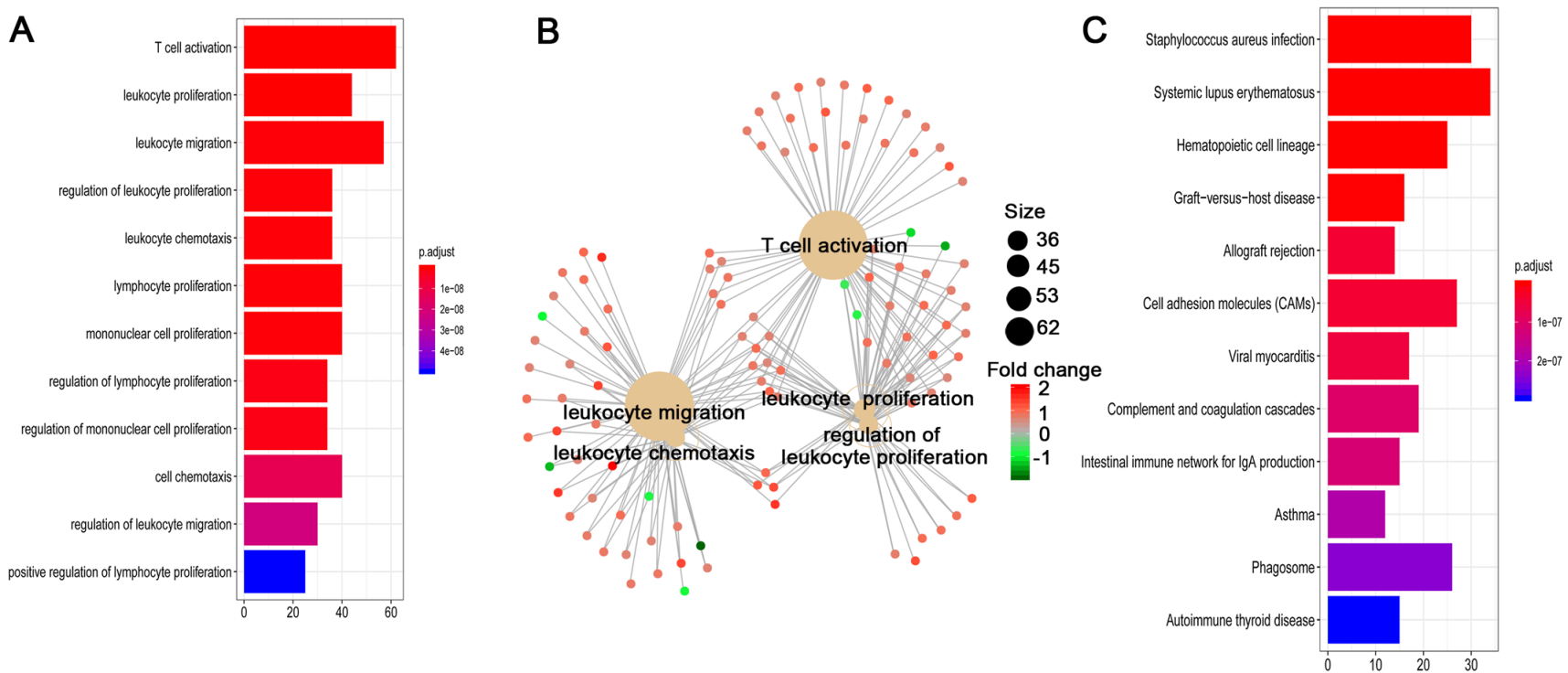

D

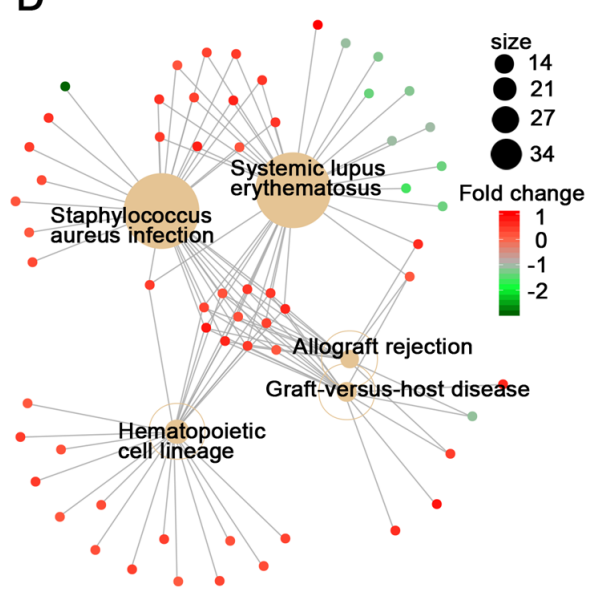

E

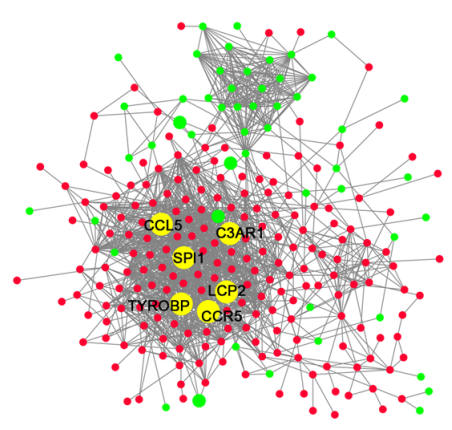

$\mathrm{F}$

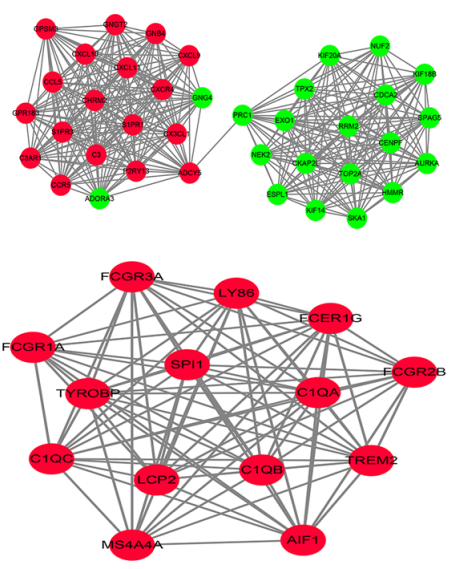

Figure 3. Gene set enrichment analysis and PPI analysis. (A) The top $10 \mathrm{GO}$ enrichment terms for the 1,050 DEGs. The original P-value was transformed to '- $\log (\mathrm{P}$-value)' to plot the curve. (B) The top 5 GO enrichment terms with their gene linkages. (C) The top 10 enriched KEGG pathways for the 1,050 DEGs. (D) The top 5 KEGG pathways with their gene linkages. (E) Construction of the PPI network of the top 400 DEGs associated with Helicobacter pylori-related gastric cancer. The big nodes represented the hub genes. (F) The top 2 sub-modules from the PPI network. PPI, protein-protein interaction; GO, Gene Ontology; DEGs, differentially expressed genes; KEGG, Kyoto Encyclopedia of Genes and Genomes.

the expression levels of PAX2, LHX3, miRNA-144-3p, miRNA-592 were downregulated, while that of ZEB1 was upregulated, which was consistent with the screening results. Furthermore, western blot analysis was used to determine the protein expression levels of the associated proteins in the normal (HP(-) tissues, as well as the tumor groups, and the clinicopathological information of the patients with gastric cancer is shown in Table SI. LHX3 was found to be downregulated and ZEB1 was upregulated, while there was no difference in PAX2 expression levels (Fig. 6H). To verify whether ZEB1 binds to the promoter region of miR-144-3p, to suppress transcriptional activity, the JASPAR database was used to predict the binding region of the miRNA-144, which was subsequently amplified and cloned into the luciferase reporter vector (Fig. 6I). As shown in Fig. 6J, ZEB1 knockdown enhanced the luciferase activity, while the overexpression of ZEB1 markedly inhibited transcriptional activity in the presence of the wild-type E-box construct only, which confirmed that ZEB1 directly targeted the E-box of the miR-144-3p gene promoter to trigger its expression. In order to further investigate whether there was significant regulatory association between ZEB1 and miR-144-3p, an siRNA for ZEB1 (siZEB1) and an overexpression plasmid (ZEB1-OE) for ZEB1 were designed and applied to attenuate or increase the expression of ZEB1 (Fig. 6J). Fig. 6K shows that miR-144-3p expression was significantly decreased in ZEB1 overexpression cells, while being significantly increased in ZEB1 knockdown cells, which further confirmed the regulatory relationship between ZEB1 and miR-144-3p. Additionally, Fig. 6L shows that luciferase activity in the WT miR-144-3p promoter was upregulated under ZEB1 deficiency, while the opposite result was observed when ZEB1 was overexpressed. No significant difference was observed when the binding site was mutated. After silencing and overexpressing PAX2 (Fig. 6M), it was found that there was no significant regulatory association between PAX2 and miR-592 (Fig. 6N). In addition, the results of the luciferase reporter assay showed that there was no significant transcriptional regulation between PAX2 and miR-592 (Fig. 6O). 
A

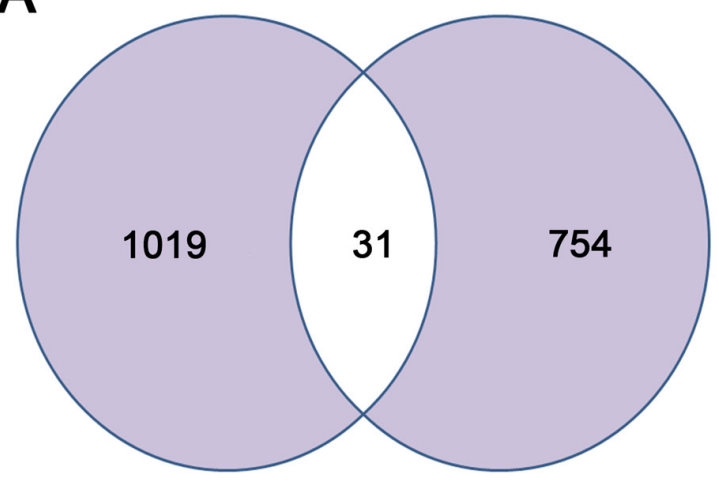

C

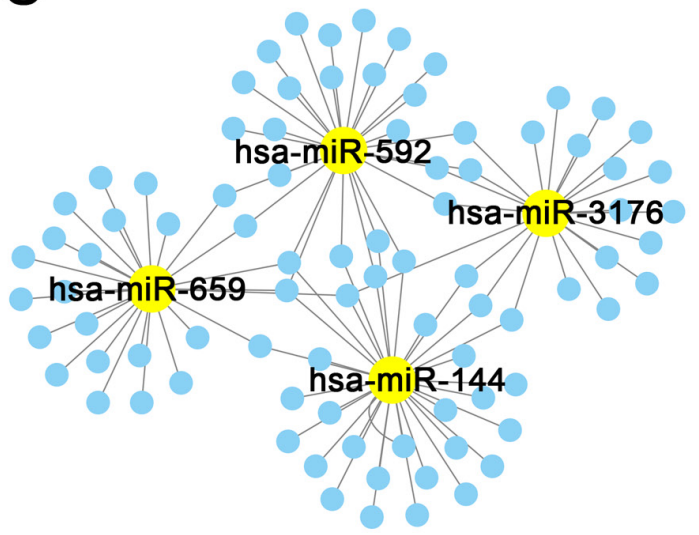

B

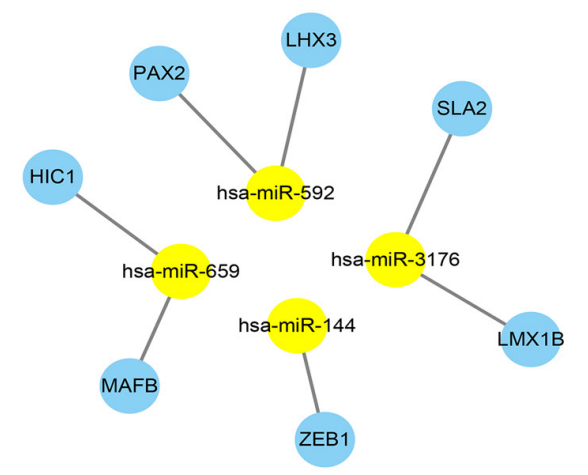

D

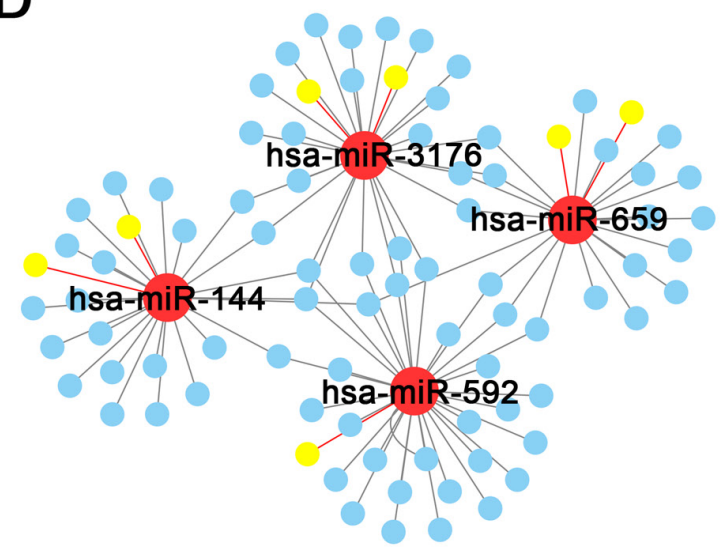

Figure 4. (A) A Venn diagram illustrating the overlap between the 1,050 DEGs and 785 TFs from the Trrust database. (B) A total of 7 TF-miRNA interactions were found. The blue and yellow nodes represent the TFs and their targeted miRNAs, respectively. (C) The 107 miRNA-DEG interactions were constructed. The yellow and blue nodes represent the miRNA and their targeted DEGs, respectively. (D) The 181 TF-miRNA-DEG networks are shown. Yellow, red and blue nodes represent the TFs, miRNAs and DEGs, respectively. TF, transcription factor; miRNA, microRNA; DEGs, differentially expressed genes.

\section{Discussion}

HP, a type-1 carcinogen, serves as the primary cause of gastric cancer (30). In 2008, 32.5\% of new cancer cases, caused by a particular infectious agent, were attributed to HP globally (31). However, the precise pathological mechanism of HP-associated gastric cancer remains unclear. Therefore, identification of the mechanisms of HP-associated gastric cancer is required to prevent progression and improve survival times. In recent years, high-throughput technology has been widely utilized to investigate gene expression levels in various types of tumor, such as colorectal (32) and ovarian cancer (33), providing a novel method to identify significant genes and determine their effects on tumor progression and initiation $(34,35)$.

In the present study, 1,050 DEGs were identified between $\mathrm{HP}(+)$ and $\mathrm{HP}(-)$ groups based on TCGA stomach cancer mRNA dataset, including 418 downregulated and 632 upregulated genes. Subsequently, enrichment and PPI analyses were used to identify the potential functions of these key genes. The top 400 DEGs, out of the total 1,050, were selected for PPI network analysis and the top key 6 genes (TYROBP, CCR5, C3AR1, LCP2, CCL5 and SPI1) were associated with HP(+) and HP(-). TYROBP and CCR5 were identified as upregulated genes by integrative analysis, which indicated that the expression of TYROBP and CCR5 could be increased in HP-associated gastric cancer. A previous study revealed that gene co-expression networks identified TREM2 and TYROBP as major hubs in human APOE expressing mice following traumatic brain injury (36). TYROBP may also play a key role in the formation of chronic gastritis and gastric ulcers. One study suggested that the inhibition of the CCL5/CCR5 axis inhibited the progression of gastric cancer (37). Thus, CCR5 may play a significant role in the tumorigenesis of gastric cancer associated with HP.

In the present study, TFs from the DEGs were subsequently identified using the Trrust database. Typically, not all DEGs corresponded to TFs. A total of 31 TFs were identified by a series of comprehensive analyses. Recently, numerous studies (38-40) have demonstrated that TFs regulate gene expression by interacting with miRNAs. Thus, the associations between the miRNAs and TFs were further investigated using the miRWalk database. Next, a TF-miRNA-target gene network was constructed to identify the potential molecular mechanisms. It was found that the ZEB1-miR-144-3p and PAX2-miR-592 networks might play key roles in the different mechanisms of $\mathrm{HP}(+)$ and $\mathrm{HP}(-)$ gastric cancer. A previous study found that ZEB1 could activate the hsa-miR-99b/let-7e/miR-125a cluster and promote 
Table III. Regulatory relations of 181 TF-miRNA-differentially expressed genes.

\begin{tabular}{|c|c|c|}
\hline TF & miRNA & Target gene \\
\hline HIC1 & hsa-miR-659 & $\begin{array}{l}\text { ADCY5, CRHR1, CYP1B1, DPYSL3, KCNMA1, MYH11, PRKG1, SFRP1, SLIT2, TLR7, } \\
\text { GPR88, CYS1, NRK, C7, HIC1, KCNC1, MYLK, NFATC4, CXCL12, USH2A, SDPR, MPDZ, } \\
\text { MAFB, CD96, SMOC2, TNFAIP8L2, MACC1 }\end{array}$ \\
\hline LHX3 & hsa-miR-592 & $\begin{array}{l}\text { C7, CYP1B1, PAX2, CDK14, DAAM2, PPP1R16B, GREM1, HAVCR2, SHE, PODN, ZCCHC24, } \\
\text { CXCL9, PFKFB2, RAG2, TOP2A, USH2A, LHX3, MS4A4A, RHPN2, GIMAP8, CYS1, SKA1 }\end{array}$ \\
\hline LMX1B & hsa-miR-3176 & $\begin{array}{l}\text { CD6, STOM, KCNC1, LMX1B, RAC2, CXCL12, TIMP3, TLL1, CD300A, RASD2, GLIPR2, } \\
\text { ZBTB7C, CD4, CRHR1, FKBP5, MYH11, PCOLCE, SLC22A14, CELF2, ADAP1, LZTS1, } \\
\text { PPP1R16B, RGMA, SLA2, GLIS2 }\end{array}$ \\
\hline MAFB & hsa-miR-659 & $\begin{array}{l}\text { ADCY5, CRHR1, CYP1B1, DPYSL3, KCNMA1, MYH11, PRKG1, SFRP1, SLIT2, TLR7, } \\
\text { GPR88, CYS1, NRK, C7, HIC1, KCNC1, MYLK, NFATC4, CXCL12, USH2A, SDPR, MPDZ, } \\
\text { MAFB, CD96, SMOC2, TNFAIP8L2, MACC1 }\end{array}$ \\
\hline PAX2 & hsa-miR-592 & $\begin{array}{l}\text { C7, CYP1B1, PAX2, CDK14, DAAM2, PPP1R16B, GREM1, HAVCR2, SHE, PODN, ZCCHC24, } \\
\text { CXCL9, PFKFB2, RAG2, TOP2A, USH2A, LHX3, MS4A4A, RHPN2, GIMAP8, CYS1, SKA1 }\end{array}$ \\
\hline SLA2 & hsa-miR-3176 & $\begin{array}{l}\text { CD6, STOM, KCNC1, LMX1B, RAC2, CXCL12, TIMP3, TLL1, CD300A, RASD2, GLIPR2, } \\
\text { ZBTB7C, CD4, CRHR1, FKBP5, MYH11, PCOLCE, SLC22A14, CELF2, ADAP1, LZTS1, } \\
\text { PPP1R16B, RGMA, SLA2, GLIS2 }\end{array}$ \\
\hline ZEB1 & hsa-miR-144-3p & $\begin{array}{l}\text { CAV2, EFEMP1, MAP1B, CXCL11, CXCL12, SFRP1, ZEB1, KIF14, AKT3, CELF2, PPP1R16B, } \\
\text { RAB9B, NAV3, ANTXR2, SHE, AXL, DSG2, CXCL9, MSN, NAP1L3, TNS1, SDPR, MPDZ, } \\
\text { LEFTY1, CPED1, CHRM2, KCNC1, NFATC4, DAAM2, APOLD1, BOC, CYP2U1 }\end{array}$ \\
\hline
\end{tabular}

$\mathrm{TF}$, transcription factor; miRNA/miR, microRNA.
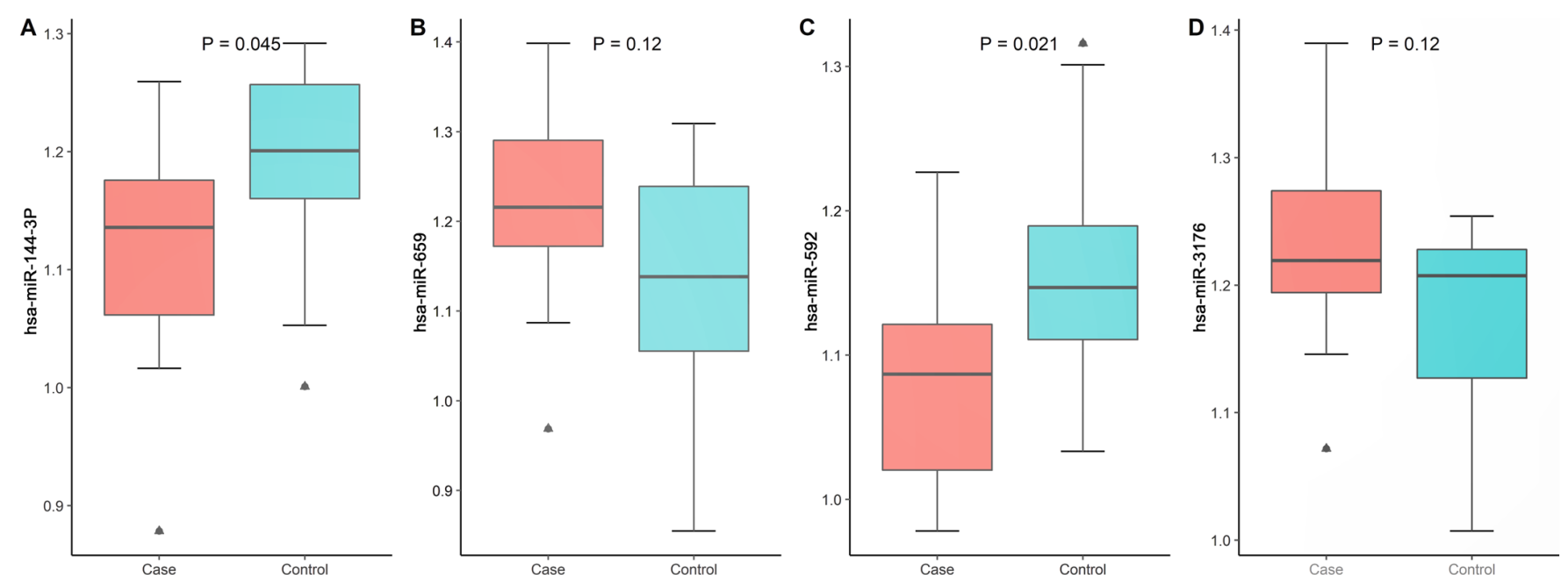

Figure 5. Reverse transcription-quantitative PCR confirmation of the expression levels of (A) hsa-miR-144, (B) hsa-miR-659, (C) hsa-miR-592 and (D) hsa-miR-3176 in Helicobacter pylori-associated gastric cancer. Data are presented as the mean \pm SD of at least three independent experiments, and statistical comparisons are case vs. control. The term 'case' represents gastric cancer HP-positive samples and the term 'control' represents gastric cancer HP-negative samples. miR, microRNA.

the invasion of liver cancer cells (41). ZEB1 may promote the invasion of liver cancer cells by regulating miR-144-3p. In addition, miR-144-3p has been shown to inhibit tumor proliferation and invasion (42). On the other hand, several studies reported that miR-144 regulated ZEB1 expression directly (43-47) and no studies explored the role of ZEB1 on miR-144-5p. No studies have shown a regulatory association between PAX2 and miR-592. In the present study, ZEB1 was identified as an upregulated gene in the $\mathrm{HP}(+)$ group, which was confirmed using RT-qPCR. Thus, ZEB1-miR-144-3p may play a key role in the pathogenesis of gastric cancer associated with HP. PAX2 was shown to play a significant role in cancer cell invasion (42). PAX2 was identified as a downregulated gene using integrative analysis. This suggested that the downregulation of PAX2 may play a significant role in the pathogenesis of gastric cancer associated with HP, by regulating miR-592. In the present study, the ZEB1-miR-144-3p was hypothesized to play key roles in the mechanism of gastric cancer associated with HP (+), which has not been previously investigated. Thus, RT-qPCR was used to confirm 

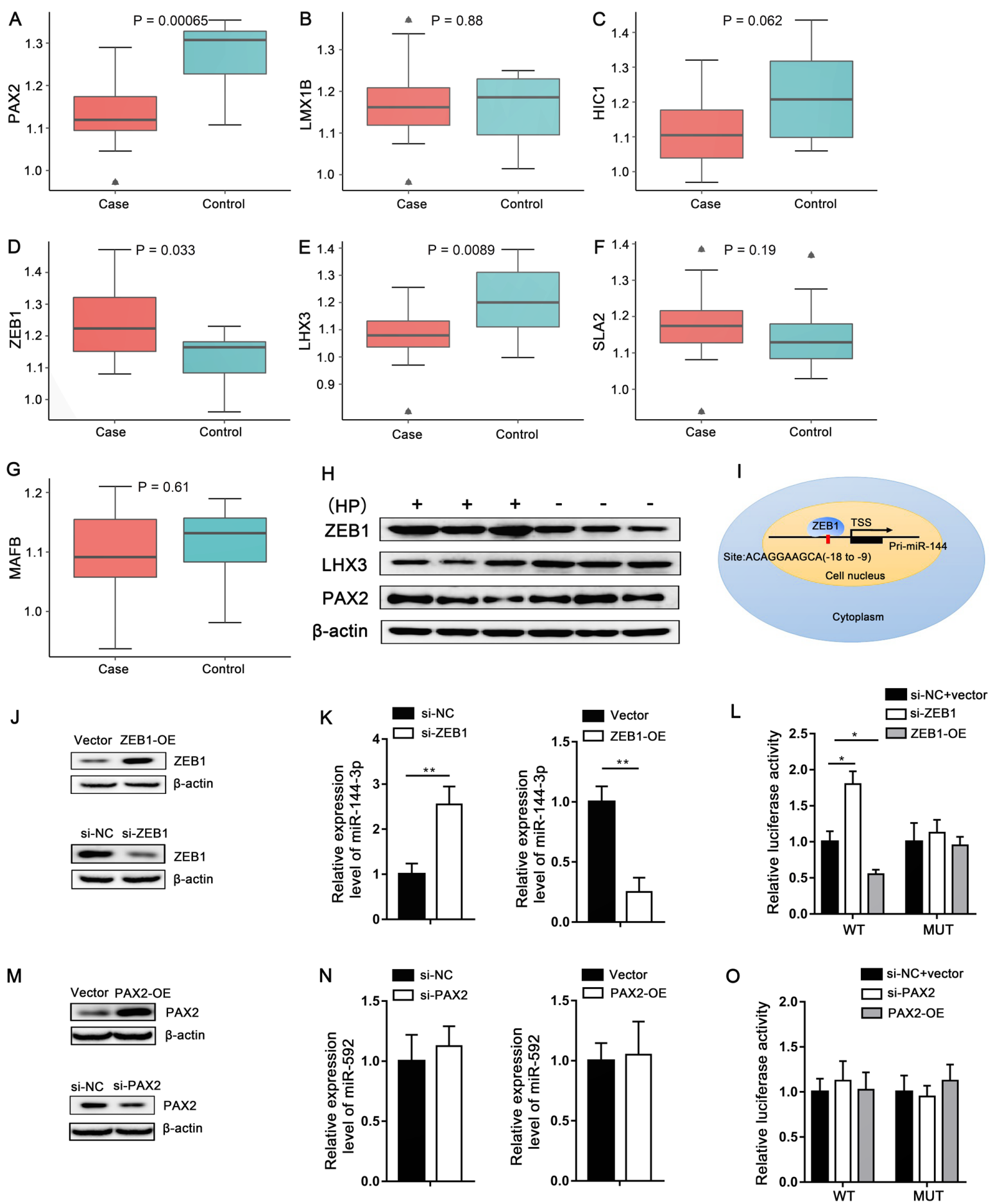

Figure 6. RT-qPCR was used to analyze the expression levels of (A) PAX2, (B) LMX1B, (C) HIC1, (D) ZEB1, (E) LHX3, (F) SLA2 and (G) MAFB in HP-associated gastric cancer. $\mathrm{HP}(+)$ is represented by the case group, while $\mathrm{HP}(-)$ is represented by the control group. (H) Western blot confirmation of the ZEB1, LHX3 and PAX2 protein expression levels in gastric cancer tissue and $\beta$-actin was used as the internal control. (I) Schematic construction of the luciferase reporter constructs containing the wild-type or mutated miR-144 gene promoter region. ( $\mathrm{J}$ and $\mathrm{K}$ ) After transfection with pcDNA-ZEB1 plasmid (ZEB1-OE) or si-ZEB1, as well as their own respective negative controls, the expression of ZEB1 and miR-144-3p in MKN-45 cells was measured by western blotting and RT-qPCR. (L) Luciferase activity assays were also performed in those treated MKN-45 cells, which were further transfected with miR-144-3p-MUT or miR-144-3p-WT. After 48 h, firefly luciferase activity was detected and normalized by Renilla luciferase activity. (M and N) After transfection with pcDNA-PAX2 plasmid (PAX2-OE) or si-PAX2, as well as their own respective negative controls, the expression of PAX2 and miR-592 in MKN-45 cells was measured by western blotting and RT-qPCR. (O) Luciferase activity assays were also performed in those treated MKN-45 cells, which were further transfected with miR-592-MUT or miR-592-WT. After 48 h, firefly luciferase activity was detected and normalized by Renilla luciferase activity. Data are presented as the mean $\pm \mathrm{SD}$ of at least three independent experiments. ${ }^{*} \mathrm{P}<0.05$ and ${ }^{* * *} \mathrm{P}<0.01$. The term 'case' represents gastric cancer $\mathrm{HP}(+)$ samples and the term 'control' represents gastric cancer HP(-) samples. HP, Helicobacter pylori; HP(+)/+, HP-positive; HP(-)/-, HP-negative; si-, siRNA; miR, microRNA; RT-qPCR, reverse transcription-quantitative PCR; OE, overexpression; NC, negative control; MUT, mutant; WT, wild-type.

the results of the bioinformatics analysis and suggested that the expression of the miRNAs and key genes was consistent with the analysis. Furthermore, the ZEB1-miR-144-3p axis was confirmed using western blot analysis and luciferase 
reporter assay. However, several limitations still exist in the present study. Firstly, the ZEB1-miR-144-3p axis was verified using a gastric cancer cell line that was HP(-), due to the difficulty of obtaining the co-cultured cell lines of HP. Secondly, the validation in another gastric cancer cell line other than MKN-45 is needed. Thirdly, the data sizes of TCGA and the collected sample data were too small to be suitable for survival analysis. More samples will be collected in further studies to explore the association between HP infection and patient prognosis.

In conclusion, in the present study, a TF-miRNA-mRNA network was constructed to analyze the potential molecular mechanisms of gastric cancer associated with HP. A total of $31 \mathrm{TFs}, 6$ key genes (TYROBP, CCR5, C3AR1, LCP2, CCL5 and SPI1), 7 TF-miRNAs and 181 TF-miRNA-mRNA sets were identified. RT-qPCR, western blot analysis and a luciferase reporter assay were used to confirm the results. The present study may provide a novel direction for further experiments, and the TF-miRNA axes may be underlying targets for precision treatment.

\section{Acknowledgements}

Not applicable.

\section{Funding}

This study was supported by the National Natural Science Foundation of China (grant no. 81702397). This project supported by Hainan Province Clinical Medical Center.

\section{Availability of data and materials}

The datasets used and/or analyzed during the current study are available from the corresponding author on reasonable request.

\section{Authors' contributions}

PZ and XM designed the study, and extracted, analyzed and interpreted the data from TCGA. JC and PZ performed the experiments and wrote the manuscript. PZ, XM and JC confirm the authenticity of all the raw data. LL and HC made substantial contributions to the conception of the study and substantively revised it. All authors have read and approved the final manuscript.

\section{Ethics approval and consent to participate}

All procedures were approved by the Ethics Committee of the Union Hospital of Huazhong University of Science and Technology (Wuhan, China), and conducted according to Declaration of Helsinki principles. Prior written and informed consent was obtained from each patient or their guardians.

\section{Patient consent for publication}

Not applicable.

\section{Competing interest}

The authors declare that they have no competing interests.

\section{References}

1. Parsonnet J, Friedman GD, Vandersteen DP, Chang Y, Vogelman JH, Orentreich N and Sibley RK: Helicobacter pylori infection and the risk of gastric carcinoma. N Engl J Med 325: 1127-1131, 1991.

2. Sepulveda AR: Helicobacter, inflammation, and gastric cancer. Curr Pathobiol Rep 1: 9-18, 2013.

3. Bartel DP: MicroRNAs: Target recognition and regulatory functions. Cell 136: 215-233, 2009.

4. Guo LL, Song CH, Wang P, Dai LP, Zhang JY and Wang KJ: Competing endogenous RNA networks and gastric cancer. World J Gastroenterol 21: 11680-11687, 2015.

5. Baer C, Claus R and Plass C: Genome-wide epigenetic regulation of miRNAs in cancer. Cancer Res 73: 473-477, 2013.

6. Singh R and Mo YY: Role of microRNAs in breast cancer. Cancer Biol Ther 14: 201-212, 2013.

7. Song S and Ajani JA: The role of microRNAs in cancers of the upper gastrointestinal tract. Nat Rev Gastroenterol Hepatol 10: 109-118, 2013.

8. O'Connell RM, Rao DS, Chaudhuri AA and Baltimore D: Physiological and pathological roles for microRNAs in the immune system. Nat Rev Immunol 10: 111-122, 2010.

9. Shrestha S, Hsu SD, Huang WY, Huang HY, Chen W, Weng SL and Huang HD: A systematic review of microRNA expression profiling studies in human gastric cancer. Cancer Med 3: 878-888, 2014.

10. Link A and Kupcinskas J: MicroRNAs as non-invasive diagnostic biomarkers for gastric cancer: Current insights and future perspectives. World J Gastroenterol 24: 3313-3329, 2018.

11. Liu L, Qu W and Zhong Z: Down-regulation of miR-503 expression predicate advanced mythological features and poor prognosis in patients with NSCLC. Int J Clin Exp Pathol 8: 5609-5613, 2015.

12. Latchman DS: Transcription factors: An overview. Int J Biochem Cell Biol 29: 1305-1312, 1997.

13. Hughes TR: Introduction to 'a handbook of transcription factors'. Subcell Biochem 52: 1-6, 2011.

14. Lichti J, Gallus C and Glasmacher E: Immune responses-transcriptional and post-transcriptional networks pass the baton. Trends Biochem Sci 43: 1-4, 2018.

15. Sussenbach JS, Rodenburg RJ, Scheper W and Holthuizen P: Transcriptional and post-transcriptional regulation of the human IGF-II gene expression. Adv Exp Med Biol 343: 63-71, 1993.

16. Lefkofsky HB, Veloso A and Ljungman M: Transcriptional and post-transcriptional regulation of nucleotide excision repair genes in human cells. Mutat Res 776: 9-15, 2015.

17. Zhang HM, Kuang S, Xiong X, Gao T, Liu C and Guo AY: Transcription factor and microRNA co-regulatory loops: Important regulatory motifs in biological processes and diseases. Brief Bioinform 16: 45-58, 2015.

18. Chen D, Lu T, Tan J,Zhao K, Li Y, Zhao W, Li H, Wang Q, Wang Y and Wei L: Identification of a transcription factor-microRNA network in esophageal adenocarcinoma through bioinformatics analysis and validation through qRT-PCR. Cancer Manag Res 11: 3315-3326, 2019.

19. Colaprico A, Silva TC, Olsen C, Garofano L, Cava C, Garolini D, Sabedot TS, Malta TM, Pagnotta SM, Castiglioni I, et al: TCGAbiolinks: An R/Bioconductor package for integrative analysis of TCGA data. Nucleic Acids Res 44: e71, 2016.

20. Ritchie ME, Phipson B, Wu D, Hu Y, Law CW, Shi W and Smyth GK: Limma powers differential expression analyses for RNA-sequencing and microarray studies. Nucleic Acids Res 43: e47, 2015 .

21. Yu G, Wang LG, Han Y and He QY: ClusterProfiler: An R package for comparing biological themes among gene clusters. OMICS 16: 284-287, 2012

22. Szklarczyk D, Gable AL, Lyon D, Junge A, Wyder S, Huerta-Cepas J, Simonovic M, Doncheva NT, Morris JH, Bork P, et al: STRING v11: Protein-protein association networks with increased coverage, supporting functional discovery in genome-wide experimental datasets. Nucleic Acids Res 47: D607-D613, 2019.

23. Smoot ME, Ono K, Ruscheinski J, Wang PL and Ideker T: Cytoscape 2.8: New features for data integration and network visualization. Bioinformatics 27: 431-432, 2011.

24. Dweep H, Gretz N and Sticht C: MiRWalk database for miRNA-target interactions. Methods Mol Biol 1182: 289-305, 2014. 
25. Friedman RC, Farh KK, Burge CB and Bartel DP: Most mammalian mRNAs are conserved targets of microRNAs. Genome Res 19: 92-105, 2009.

26. Betel D, Koppal A, Agius P, Sander C and Leslie C: Comprehensive modeling of microRNA targets predicts functional non-conserved and non-canonical sites. Genome Biol 11: R90, 2010.

27. Kertesz M, Iovino N, Unnerstall U, Gaul U and Segal E: The role of site accessibility in microRNA target recognition. Nat Genet 39: 1278-1284, 2007.

28. Livak KJ and Schmittgen TD: Analysis of relative gene expression data using real-time quantitative PCR and the 2(-Delta Delta C(T)) method. Methods 25: 402-408, 2001.

29. Khan A, Fornes O, Stigliani A, Gheorghe M, CastroMondragon JA, van der Lee R, Bessy A, Chèneby J, Kulkarni SR Tan G, et al: JASPAR 2018: Update of the open-access database of transcription factor binding profiles and its web framework. Nucleic Acids Res 46: D260-D266, 2018.

30. Schistosomes, liver flukes and Helicobacter pylori. IARC Working Group on the Evaluation of Carcinogenic Risks to Humans. Lyon, 7-14 June 1994. IARC Monogr Eval Carcinog Risks Hum 61: 1-241, 1994.

31. de Martel C, Ferlay J, Franceschi S, Vignat J, Bray F, Forman D and Plummer M: Global burden of cancers attributable to infections in 2008: A review and synthetic analysis. Lancet Oncol 13: 607-615, 2012.

32. Xu H, Liu Y, Cheng P, Wang C, Liu Y, Zhou W, Xu Y and Ji G: CircRNA_0000392 promotes colorectal cancer progression through the miR-193a-5p/PIK3R3/AKT axis. J Exp Clin Cancer Res 39: 283, 2020.

33. Khan K, Javed Z, Sadia H, Sharifi-Rad J, Cho WC and Luparello C: Quercetin and MicroRNA interplay in apoptosis regulation in ovarian cancer. Curr Pharm Des 27: 2328-2336, 2021.

34. Carvalho GB, Costa LE, Lage DP, Ramos FF, Santos TTO, Ribeiro PAF, Dias DS, Salles BCS, Lima MP, Carvalho LM, et al: High-through identification of T cell-specific phage-exposed mimotopes using PBMCs from tegumentary leishmaniasis patients and their use as vaccine candidates against Leishmania amazonensis infection. Parasitology 146: 322-332, 2019.

35. Steinlechner M and Parson W: Automation and high through-put for a DNA database laboratory: Development of a laboratory information management system. Croat Med J 42: 252-255, 2001

36. Castranio EL, Mounier A, Wolfe CM, Nam KN, Fitz NF, Letronne F, Schug J, Koldamova R and Lefterov I: Gene co-expression networks identify Trem 2 and Tyrobp as major hubs in human APOE expressing mice following traumatic brain injury. Neurobiol Dis 105: 1-14, 2017.

37. Aldinucci D and Casagrande N: Inhibition of the CCL5/CCR5 Axis against the progression of gastric cancer. Int J Mol Sci 19: 1477,2018
38. Bo C, Zhang H, Cao Y, Lu X, Zhang C, Li S, Kong X, Zhang X, Bai M, Tian K, et al: Construction of a TF-miRNA-gene feed-forward loop network predicts biomarkers and potential drugs for myasthenia gravis. Sci Rep 11: 2416, 2021.

39. Hong Z, Wang Q, Hong C, Liu M, Qiu P, Lin R, Lin X, Chen F, Li Q, Liu L, et al: Identification of seven cell cycle-related genes with unfavorable prognosis and construction of their TF-miRNA-mRNA regulatory network in breast cancer. J Cancer 12: 740-753, 2021.

40. Bertonha FB, Bando SY, Ferreira LR, Chaccur P, Vinhas C, Zerbini MC, Carneiro-Sampaio MM and Moreira-Filho CA: Age-related transcriptional modules and TF-miRNA-mRNA interactions in neonatal and infant human thymus. PLoS One 15: e0227547, 2020

41. Wang XF, Liu JY, Li JL, Wang FZ and Sun BL: ZEB1 causes the production of hsa-microRNA-99b/let-7e/microRNA-125a cluster and promotes invasion of liver cancer cells. Eur Rev Med Pharmacol Sci 23: 1468-1475, 2019.

42. Cao J, Han X, Qi X, Jin X and Li X: TUG1 promotes osteosarcoma tumorigenesis by upregulating EZH2 expression via miR-144-3p. Int J Oncol 51: 1115-1123, 2017.

43. Guan H, Liang W, Xie Z, Li H, Liu J, Liu L, Xiu L and Li Y: Down-regulation of miR-144 promotes thyroid cancer cell invasion by targeting ZEB1 and ZEB2. Endocrine 48: 566-574, 2015.

44. Pan Y, Zhang J, Fu H and Shen L: MiR-144 functions as a tumor suppressor in breast cancer through inhibiting ZEB1/2-mediated epithelial mesenchymal transition process. Onco Targets Ther 9: 6247-6255, 2016

45. Zhang G, An H and Fang X: MicroRNA-144 regulates proliferation, invasion, and apoptosis of cells in malignant solitary pulmonary nodule via zinc finger E-box-binding homeobox 1. Int J Clin Exp Pathol 8: 5960-5967, 2015.

46. Pan HL, Wen ZS, Huang YC, Cheng X, Wang GZ, Zhou YC, Wang ZY, Guo YQ, Cao Y and Zhou GB: Down-regulation of microRNA-144 in air pollution-related lung cancer. Sci Rep 5: $14331,2015$.

47. Gao ZY, Liu H and Zhang Z: MiR-144-3p increases radiosensibility of gastric cancer cells by targeting inhibition of ZEB1. Clin Transl Oncol 23: 491-500, 2021.

48. Wittekind C: The development of the TNM classification of gastric cancer. Pathol Int 65: 399-403, 2015.

(i) $\odot$ This work is licensed under a Creative Commons Attribution-NonCommercial-NoDerivatives 4.0 International (CC BY-NC-ND 4.0) License. 